
\title{
28 Research Square \\ C-Reactive Protein and Lymphocyte are important indicators of severe coronavirus disease and poor prognosis in elderly patients
}

\section{Qinglin He}

School of Medicine,Wuhan University of Science and Technology

\section{Xiafen $\mathrm{Hu}$}

School of Medicine,Wuhan University of Science and Technology

\section{Xiaochen Xiang}

School of Medicine,Wuhan University of Science and Technology

\section{Siyang Chen}

School of Medicine,Wuhan University of Science and Technology

Wanxin Liu

School of Medicine,Wuhan University of Science and Technology

Xiaoliu Liu

School of Medicine,Wuhan University of Science and Technology

\section{Qiang Wang ( $\nabla$ wangqiang@wust.edu.cn )}

https://orcid.org/0000-0001-7420-8646

\section{Kaiwen Guo}

School of medicine,Wuhan University of Science and Technology

\section{Qingming Wu}

School of Medicine,Wuhan University of Science and Technology

\section{Research article}

Keywords: COVID - 19, Elderly patients, CRP, L, Severity, Prognosis

Posted Date: August 13th, 2020

DOI: https://doi.org/10.21203/rs.3.rs-21426/v3

License: (c) (i) This work is licensed under a Creative Commons Attribution 4.0 International License. Read Full License 


\section{Abstract}

Objective区To explore the value of C-reactive protein (CRP) and lymphocyte (L) in the assessment of disease severity and prognosis of elderly COVID-19 patients.

Methods: A total of 194 positive COVID-19 patients were collected from Tianyou Hospital and Puren Hospital, affiliated hospital of Wuhan University of Science and Technology. Their demographic characteristics were analyzed. The dynamic changes of CRP and $L$ in peripheral blood were retrospectively studied.

Results: (1) There were significant statistical differences in CRP, L in clinical typing and clinical outcome in patients over 60 years old compared with those under 60 years old. Survival analysis showed that the risk of death was greater in patients over 60 than in those under $60 . \otimes 2$ In 125 patients over 60 years old, the hospitalized patients with severe or critical types of disease had significantly higher CRP than those with moderate type $(p<0.01)$. In the outcome of the elderly patients, the CRP of the patients with the outcomes of discharge, improvement, aggravation and death increased successively $(p<0.01)$. According to the analysis of Logistic regression model, the increase of CRP constitutes a risk factor for death in elderly patients. (3) In the ROC curve analysis to distinguish the death outcome and non-death outcome of COVID-19 patients, the area under the curve (AUC) of CRP and L was 0.751 and 0.720 respectively. CRP and $L$ had good diagnostic accuracy for the death outcome of patients. (4) Changes in CRP were correlated with changes in CT imaging and were consistent with changes in the course of the disease.

Conclusions: (1) The data collected in this research showed that the cumulative survival rate of patients over 60 years old was lower than that of patients under 60 years old. With the increase of age, the CRP of patients showed an increasing trend, and the $L$ of patients showed a characteristic lower than the normal reference interval. (2) CRP and $L$ are important monitoring indicators of COVID-19 in elderly patients. Combined with CT examination and observation of their dynamic changes, CRP and L are of important clinical guiding value for the judgment of disease severity and the evaluation of prognosis.

\section{Introduction}

Since December 2019, novel coronavirus (SARS-CoV-2) has been identified as the cause of pneumonia in Wuhan, Hubei province, China ${ }^{[1-2]}$. The World Health Organization has officially named the Disease caused by SARS-CoV-2 as Corona Virus Disease (COVID-19) ${ }^{[3-4]}$. At present, COVID - 19 has been effectively controlled in China, but there is still a global outbreak, which has become a global public health problem. SARS-CoV-2 spreads rapidly, causing a wide range of lesions with varying degrees of severity. However, the condition of some elderly patients changes rapidly, and inflammatory storms rapidly appear, leading to worsening or even life-threatening conditions ${ }^{\text {[5-8] }}$. Therefore, early diagnosis of the severity of the disease and prognosis of the elderly patients are particularly important. Absolute values of C-reactive protein (CRP) and lymphocyte (L), commonly used laboratory inflammatory indicators in clinical practice, C-reactive protein (CRP) is a protein (acute protein) that rises sharply in the plasma when the body is infected or damaged. Lymphocyte $(\mathrm{L})$ count refers to the number of lymphocytes in the peripheral blood. The normal range of lymphocyte count measured in this study is $1.1 \times 109$ / L-3.2 $\times 109 / \mathrm{L}$, which is abnormal if it is lower or higher than the normal range. These indicators can assist in the diagnosis of infectious inflammation and disease severity in patients, but are they also applicable to elderly patients with COVID-19? And can we determine the early prognosis? In our previous studies ${ }^{[9]}$, we have concluded that COVID - 19 patients with Serum amyloid A (SAA), CRP levels increased significantly, $L$ is on the decline, as the disease progression, from light, heavy to the critically ill, SAA, CRP gradually increases, while $L$ gradually decline, but now we find in between the 
elderly and young patients with the disease and prognosis of differences, therefore, this study explore the C-reactive protein and lymphocyte count in aged > 60 years COVID - 19 patients with disease severity and prognosis evaluation of value. Now, we continue to increase the sample size by about $50 \%$ based on the previous study. We retrospectively analyzed the demographic characteristics of 194 COVID-19 patients in a designated COVID-19 hospital in Wuhan, China, we studied the dynamic changes of inflammatory indicators CRP and $L$ in peripheral blood of 125 patients over 60 years old, and explored the dynamic changes of inflammatory indicators in peripheral blood of elderly patients with COVID-19, so as to determine whether it can be used to determine the severity and prognosis of COVID-19 in elderly patients.

\section{Materials And Methods}

\section{Case source and inclusion criteria}

This study was approved by the Medical Ethics Review Board of Wuhan University of Science and Technology (No. 202009).

1) All cases came from Tianyou Hospital and Puren Hospital, affiliated Hospital of Wuhan University of Science and Technology from January 18, 2020 to February 26, 2020, tested for SARS-CoV-2 pathogenic nucleic acid Positive hospitalized cases;

2) Completed the laboratory data collection and clinical condition assessment at the three time points required by this study;

3) Study composite endpoint cures discharged patients and deaths before February 26, 2020; cases being treated in hospital on February 26, 2020.

\section{Exclusion criteria}

Those who failed to collect laboratory data in three times.

3. Criteria for evaluation of clinical conditions

1) Case classification

According to the COVID-19 diagnosis and treatment plan issued by the National Health Commission of China, it is divided into 4 types: mild, moderate, severe and critical. In the analysis of this study, the patients of mild type were merged into moderate type.

Mild type: mild clinical symptoms, no pneumonia on imaging

Moderate type: Symptoms such as fever, respiratory tract, pneumonia can be seen on imaging.

Severe type: According with any of the following.

(1) Shortness of breath, RR>30 Times / minute.

(2) At rest, Oxygen saturation $\leq 93 \%$. 
(3) $\mathrm{PaO}_{2} / \mathrm{FiO}_{2} \leq 30 \mathrm{mmHg} \otimes 1 \mathrm{mmHg}=0.133 \mathrm{kPa} \otimes$, Pulmonary imaging showed significant progression of $>50 \%$ within 24 to 48 hours.

Critical type: One of the following conditions:

(1) Have respiratory failure and require mechanical ventilation;

(2) Shock;

(3) Combining other organ failure requires ICU monitoring treatment.

This study mainly studied the demographic characteristics, disease severity and outcome of COVID-19 patients. To optimize the research program, we mixed light and common types into light and common types according to the research purpose and clinical practice.

2) Clinical outcome

According to the clinical characteristics, the patients were divided into four types: discharge, improvement, aggravation and death.

\section{Treatment method}

According to the COVID-19 diagnosis and treatment plan issued by the National Health Commission of China, patients generally receive effective oxygen therapy, lopinavir / ritonavir and other drug antiviral treatments, and provide respiratory and circulatory support treatment for patients with severe and critical illness.

5. Composite endpoints of the study

By February 26, 2020, the number of discharge and death cases before February 26, 2020 should be counted, and the length of stay in the hospital should be more than 7 days. The changes of their conditions should be evaluated, and the outcome indicators should be improved, aggravated and stable.

6. Experimental data collection

The experimental data were collected at three time points: admission, 3-5 days of hospitalization, and the composite end point. At the same time, clinical typing, disease severity and outcome were determined, and the corresponding length of hospitalization was collected.

The specific methods of blood routine test and CRP test were as follows:

1) The venous blood of the subjects was extracted in the fasting state of the patients in the morning, and the venous blood was placed in a $2 \mathrm{ml}$ EDTA-K2 anticoagulant tube and a $5 \mathrm{~mL}$ procoagulant tube respectively, mixed upsidedown, and sent for timely examination.

$2 \llbracket 2 \mathrm{ml}$ EDTA-K2 anticoagulant whole blood samples were put into automatic hemocyte analyzer for routine blood test.

$3 \otimes$ The $5 \mathrm{ml}$ procoagulant tube was put into an ordinary low-speed centrifuge and centrifuge at $3000 \mathrm{rpm} / \mathrm{min}$ for $10 \mathrm{~min}$. After the serum was separated, the sample was put into a biochemical analyzer and the CRP level in the blood samples was detected by immunoturbidimetry. 
4) The normal reference range of $\mathrm{CRP}$ is $<3 \mathrm{mg} / \mathrm{L}$; The normal reference range of lymphocytes is $1.1 \times 10^{9} / \mathrm{L}-3.2 \times$ $10^{9} / \mathrm{L}$.

\section{Analytical methods}

SPSS25.0 software was used for statistical analysis. Non-parametric test (Mann-Whitney test) was used for quantitative data with skewed distribution and uneven variance to analyze differences between groups, and chisquare test was used for qualitative data to analyze differences between groups, if the theoretical frequency is less than 1 , the Fisher exact probability test method is adopted. Survival analysis was used to determine the difference in survival time distribution of patients under different conditions during clinical observation. The risk degree of admission type and death outcome of each factor was analyzed by binary regression model and multiple regression model. The diagnostic value of CRP, $L$ and other indicators on the death outcome was analyzed by the receiver operating characteristic curve (ROC), and the area under the curve (AUC) and its 95\% Cl were calculated. The optimal threshold value is determined by the maximum approximate index, providing both sensitivity and specificity. The test level selected for this statistical analysis was $\mathrm{a}=0.05$, and $\mathrm{P}<0.05$ indicated that the difference was statistically significant.

\section{Results}

The 194 patients included in this research were divided into two groups, younger age group(<60 years old) and older age group $\varangle \geq 60$ years old $\nabla$. Among them, older age group (125 patients) were more than younger age group (69 patients). The $\mathrm{M} \pm \mathrm{SD}$ of Hospitalization days, C-reactive protein, lymphocyte count were calculated. CRP and $\mathrm{L}$ were grouped according to the normal reference range (CRP: $<3 \mathrm{mg} / \mathrm{L}, 3-100 \mathrm{mg} / \mathrm{L},>100 \mathrm{mg} / \mathrm{L} ; \mathrm{L}: 1.1-3.2 \times 10^{9} / \mathrm{L},<1.1 \times$ $10^{9} / \mathrm{L},>3.2 \times 109$ / L). For quantitative data, the average of Hospitalization days in the two age groups was 20.71 days and 20.27 days, respectively. The average CRP level of older age group is higher than younger age group, with mean values of 55.06 and 39.59. The average $L$ level of younger age group is closer to the normal reference range than older age group. In the classified data, $90.21 \%$ of patients were above the normal level of CRP and $34.54 \%$ of patients had $L$ levels deviated from the normal range. By difference test (normally distributed data: Student's $t$ test; The non-normal distribution data: Mann-Whitney $U$ test) showed that there was no significant statistical difference in the hospitalization days between the two groups of patients of different ages, the significance of CRP was close to $p=0.05$ in the two groups, and there was a significant statistical difference in $L$ between the two groups $(p<$ 0.05). (See Table 1).

Table 1 Demographic characteristics of the patient

\begin{tabular}{|c|c|c|c|c|}
\hline & \multicolumn{2}{|c|}{ Age stratification } & \multirow[b]{2}{*}{$\begin{array}{c}\text { Total } \\
\square \mathrm{n}=194 \square\end{array}$} & \multirow[b]{2}{*}{ P value } \\
\hline & $\begin{array}{c}<60 \\
\square n=69 \square\end{array}$ & $\begin{array}{c}\geq 60 \\
\square n=125 \square\end{array}$ & & \\
\hline HOD & $20.71 \pm 6.63$ & $20.27 \pm 6.27$ & $20.43 \pm 6.39$ & $0.823^{\mathrm{a}}$ \\
\hline CRP & $39.59 \pm 47.15$ & $55.06 \pm 49.72$ & $49.56 \pm 49.26$ & $0.061^{\mathrm{b}}$ \\
\hline $\begin{array}{l}<3 \\
3-100 \\
>100\end{array}$ & $\begin{array}{c}14(7.22 \%) \\
46(23.71 \%) \\
9(4.64 \%)\end{array}$ & $\begin{array}{c}5(2.58 \%) \\
93(47.94 \%) \\
27(13.92 \%)\end{array}$ & $\begin{array}{c}19(9.79 \%) \\
139(71.65 \%) \\
36(18.56 \%)\end{array}$ & \\
\hline $\mathrm{L}$ & $1.87 \pm 3.60$ & $0.91 \pm 0.59$ & $1.25 \pm 2.24$ & $0.010^{\mathrm{b}}$ \\
\hline $\begin{array}{l}<1.1 \\
1.1-3.2 \\
>3.2\end{array}$ & $\begin{array}{c}32(16.49 \%) \\
34(17.53 \%) \\
3(1.55 \%)\end{array}$ & $\begin{array}{c}30(15.46 \%) \\
93(47.94 \%) \\
2(1.03 \%)\end{array}$ & $\begin{array}{c}62(31.96 \%) \\
127(65.46 \%) \\
5(2.58 \%)\end{array}$ & \\
\hline
\end{tabular}

Note: HOD: Hospitalization days $\square$ Normal reference values: CRP (<3mg / L); L $\left(1.1 \times 10^{9} / \mathrm{L}-3.2 \times 10^{9} / \mathrm{L}\right)$

${ }^{\text {a }}$ Student's t test (double-tail test) $\square^{\mathrm{b}}$ Mann-Whitney U test (double-tail test) 
In Figure 1, patients were divided into moderate, severe \& critical groups. As can be seen from Figure 1, the mean values of $L$ and CRP in older age group were lower than younger age group in the Moderate group. In the Severe \& Critical group, the mean CRP of older age group was higher than younger age group, and the mean $L$ of older age group was lower than younger age group. Mann-whitney test was used to analyze the differences of CRP and L levels in patients of different ages in the two groups. In Figure 1A, Patients of different ages with Moderate clinical classification showed no significant difference in CRP $(p>0.05)$, but patients of different ages with Severe \& critical clinical classification showed significant statistical difference in CRP $(p<0.01)$. In Figure 1B, there were significant statistical differences in $L$ levels between younger age group and older age group in the Moderate and Severe \& Critical groups $(P<0.05)$.

As can be seen from Figure 1, when the disease is severe, CRP of elderly patients is higher than that of young patients, and $L$ of elderly patients is lower than that of young patients and lower than the normal reference value. Therefore, we established a linear regression model to explore whether CRP and L of COVID-19 patients show a linear change trend with age. In Figure 2, the linear regression analysis showed that the CRP of the patients increased with the increase of age, while $L$ decreased with the increase of age. It can be seen from this data that the CRP value of the elderly patients was higher while $L$ was lower. The regression equations of CRP and $L$ were respectively deduced by regression variance analysis for the population regression coefficient, and the relationship between CRP and the age of the patients was linear regression $(p<0.05)$, while there was no significant linear regression relationship between $L$ and the age of the patients $(p>0.05)$.

In Figure 3, the Kaplan-Meier method of survival analysis was used to describe the cumulative survival rate at the end of events during hospitalization. With the increase of observation time in hospital for all patients, the survival rate curve gradually showed a step-like decline from 1-0. During in-hospital clinical observation, older age group had earlier death events compared with younger age group. The log-rank test showed that $P<0.05(P=0.013)$. The survival rate of patients in the two groups was significantly different and the difference was statistically significant, so the risk of death in older age group was greater than younger age group.

In Table 2, univariate logistic regression analysis showed that age was an independent influence factor of clinical classification and clinical outcome $(P<0.05)$. younger age group had a severe clinical classification and a 0.471 times higher risk of critical type than older age group (OR=0.471ष95\% Cl: $0.259-0.856)$, and the risk of death was

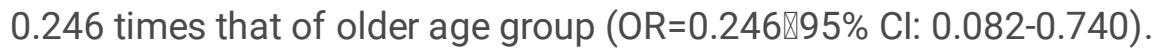

Therefore, 125 samples of elderly patients (over 60 years old) were extracted in this study and the relationship between characteristics of elderly patients (demographic characteristics, clinical indicator characteristics) and clinical classification and clinical outcome was studied.

Table 2 Univariate regression analysis of independent factors influencing Clinical classification and clinical outcome

\begin{tabular}{lcccccc}
\hline & \multicolumn{4}{c}{ Univariate Logistic regression analysis of Clinical classification } \\
\cline { 2 - 6 } & $\mathrm{B}$ & $\mathrm{SE}$ & Wald c$^{2}$ & $\mathrm{p}$ & Exp (B) & $95 \% C I$ \\
\hline Age 60 & -0.754 & 0.305 & 6.092 & 0.014 & 0.471 & $\square 0.259 \square 0.856 \square$ \\
Age $\geq 60$ & & & & & 1 & \\
\hline & Univariate Logistic regression analysis of Clinical outcome \\
\cline { 2 - 7 } & $\mathrm{B}$ & $\mathrm{SE}$ & Wald c$^{2}$ & $\mathrm{p}$ & Exp (B) & $95 \% C I$ \\
\hline Age 60 & -1.402 & 0.562 & 6.231 & 0.013 & 0.246 & $\square 0.082 \square 0.740 \square$ \\
Age $\geq 60$ & & & & & 1 & \\
\hline
\end{tabular}

In Table 3, 125 patients over 60 years old were tested with chi-square test (Fisher's Exact Test was used for theoretical frequency $T<1$ ). The statistical test results showed that the different levels of $C R P$ and $L$ were not identical with the distribution of clinical classification and clinical outcome, and there were significant statistical 
differences between the levels of CRP and $L$ and the clinical classification and clinical outcome of patients admitted to hospital $(p<0.05)$.

Table 3 Correlation between CRP and L and clinical classification and clinical outcome of the elderly over 60 years old

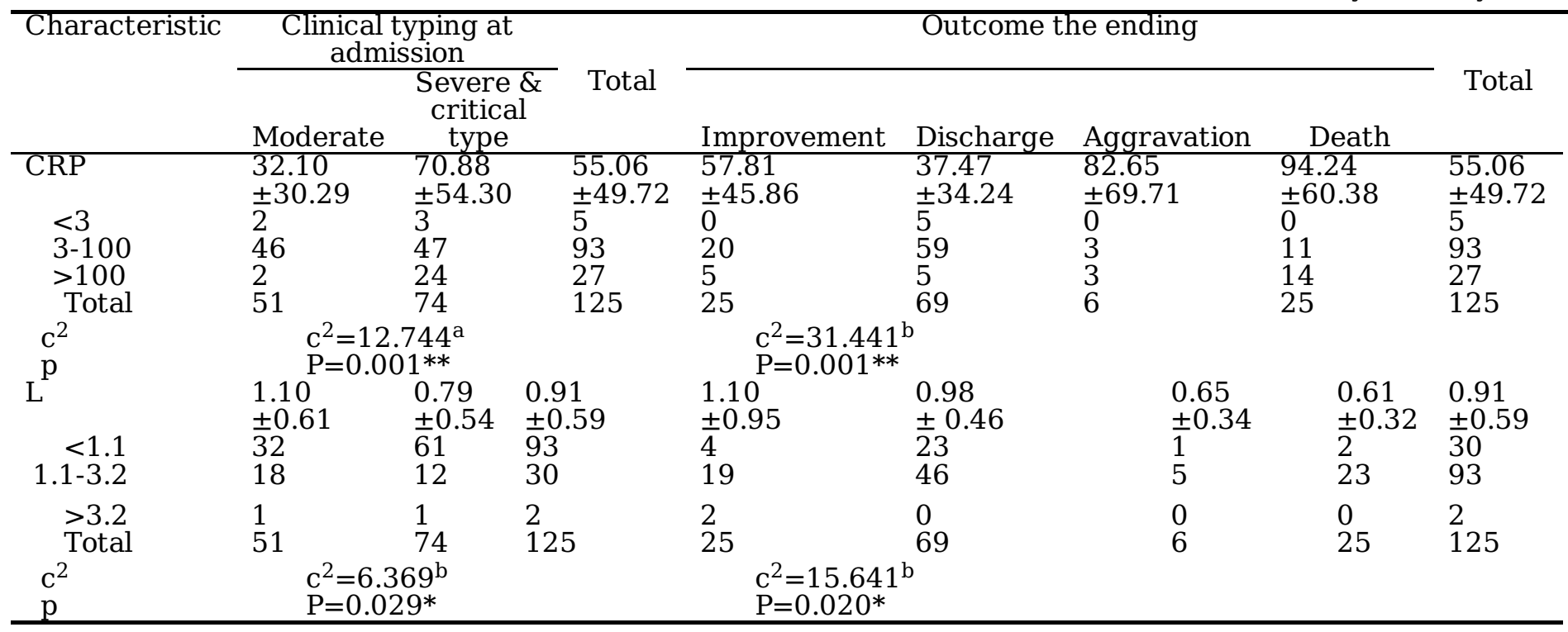

Note: Normal reference values: CRP (<3mg / L); L $\left(1.1 \times 10^{9} / \mathrm{L}-3.2 \times 10^{9} / \mathrm{L}\right)$

${ }^{\mathrm{a}}$ Chi-square test (double-tail test) $\square^{\mathrm{b}}$ Fisher's exact test(double-tail test)

*At level 0.05 (double-tailed), **At level 0.01 (double-tailed), the correlation was significant.

In the binary Logistic regression analysis of whether the elderly patients had severe or critical clinical type, we used univariate Logistic regression analysis to preliminarily determine the factors affecting clinical type and the degree of risk, and the results showed that CRP level, L number had a significant impact on the clinical type of the patients. For each unit increase of CRP index, the risk of disease severity was increased 1.022 times, and the $95 \%$ interval of OR value was greater than 1 , which constitute the conditions of risk factors. For every unit increase in $L$ level, the risk of disease severity increased by 0.359 times. In order to correct the effects of confounding factors, we will be more variable into the multi-factor Logistic regression analysis, the results showed that CRP index for every rise in unit, 1.019 times the risk of disease severity ascension, OR 95\% of the value range are greater than 1, constitute the conditions of the dangerous factors, $L$ level for every rise in unit, the risk of disease severity increase 0.478 times. (See Table 4)

Table 4 Logistic regression analysis of influencing factors of clinical classification in patients over 60 years old

\begin{tabular}{lcccccc}
\hline Factor & \multicolumn{6}{c}{ Univariate Logistic regression analysis } \\
\cline { 2 - 7 } & $\mathrm{B}$ & $\mathrm{SE}$ & Wald c$^{2}$ & $\mathrm{p}$ & $\mathrm{Exp}(\mathrm{B})$ & $95 \% C I$ \\
\hline Sex(male) & 0.650 & 0.383 & 2.881 & 0.090 & 1.915 & $\square 0.904 \square 4.053 \square$ \\
HOD & -0.042 & 0.030 & 2.006 & 0.157 & 0.959 & $\square 0.904 \square 1.016 \square$ \\
CRP & 0.021 & 0.005 & 15.844 & 0.000 & 1.022 & $\square 1.011 \square 1.032 \square$ \\
L & -1.024 & 0.396 & 6.962 & 0.008 & 0.359 & $\square 0.168 \square 0.769 \square$ \\
\hline Factor & \multicolumn{6}{c}{ Multivariate Logistic regression analysis } \\
\cline { 2 - 7 } & $\mathrm{B}$ & $\mathrm{SE}$ & Wald c & $\mathrm{p}$ & Exp(B) & $95 \% C I$ \\
\hline Sex(male) & 0.268 & 0.431 & 0.386 & 0.534 & 1.307 & $\square 0.562 \square 3.039 \square$ \\
HOD & -0.027 & 0.034 & 0.627 & 0.428 & 0.973 & $\square 0.910 \square 1.041 \square$ \\
CRP & 0.019 & 0.006 & 11.376 & 0.001 & 1.019 & $\square 1.008 \square 1.031 \square$ \\
L & -0.738 & 0.356 & 4.290 & 0.038 & 0.478 & $\square 0.238 \square 0.961 \square$ \\
\hline
\end{tabular}


Note: HOD: Hospitalization days $\square$

In the multivariate Logistic regression analysis of hospitalization days, gender, CRP, L and clinical outcomes of elderly patients, the elderly patients discharged from hospital were compared with elderly patients who died『when each unit increase in CRP, the risk of death in discharged patients was 0.981 times that of those who died. Compared with the elderly patients who died, the increased length of stay in hospital constituted the risk factor for death, and the OR value was 1.330, 1.396 and 1.377. (See Table 5)

Table 5 multivariate Logistic regression analysis of the influencing factors of mortality outcome in patients

\begin{tabular}{|c|c|c|c|c|c|c|c|}
\hline Factors & $\bar{B}$ & SE & Wald $c^{2}$ & $\bar{p}$ & $\operatorname{Exp}(\mathrm{B})$ & \multicolumn{2}{|c|}{$95 \% C I$} \\
\hline \multicolumn{8}{|l|}{ Discharged } \\
\hline & 0.285 & 0.069 & 16.854 & 0.000 & 1.330 & 1.161 & 1.524 \\
\hline CRP & -0.019 & 0.007 & 7.700 & 0.006 & 0.981 & 0.968 & 0.994 \\
\hline $\mathrm{L}$ & 2.003 & 0.943 & 4.511 & 0.034 & 7.412 & 1.167 & 47.062 \\
\hline$[\mathrm{sex}=$ male $]$ & 0.451 & 0.686 & 0.433 & 0.510 & 1.570 & 0.410 & 6.019 \\
\hline$[\mathrm{sex}=$ female $]$ & 0 & & & & & & \\
\hline \multicolumn{8}{|l|}{ Improvement } \\
\hline HOD & 0.334 & 0.076 & 19.159 & 0.000 & 1.396 & 1.202 & 1.621 \\
\hline CRP & -0.004 & 0.007 & 0.322 & 0.570 & 0.996 & 0.982 & 1.010 \\
\hline $\mathrm{L}$ & 2.477 & 0.986 & 6.314 & 0.012 & 11.907 & 1.724 & 82.215 \\
\hline$[\operatorname{sex}=$ male $]$ & -0.037 & 0.765 & 0.002 & 0.961 & 0.963 & 0.215 & 4.312 \\
\hline$[\mathrm{sex}=$ female $]$ & & & & & & & \\
\hline \multicolumn{8}{|l|}{ Exacerbation } \\
\hline HOD & 0.320 & 0.105 & 9.249 & 0.002 & 1.377 & 1.121 & 1.693 \\
\hline CRP & 0.000 & 0.010 & 0.000 & 0.991 & 1.000 & 0.981 & 1.020 \\
\hline $\mathrm{L}$ & 1.091 & 1.509 & 0.523 & 0.470 & 2.977 & 0.155 & 57.253 \\
\hline$[\operatorname{sex}=$ male $]$ & 0.941 & 1.270 & 0.549 & 0.459 & 2.563 & 0.213 & 30.901 \\
\hline$[\mathrm{sex}=$ female $]$ & 0 & & & & & & \\
\hline
\end{tabular}

Note: HOD: Hospitalization days

In the analysis of the prognostic diagnostic value of CRP and L on death outcome in elderly patients, we used nondeath outcome and death outcome as the basis for positive classification. With AUC $=0.5$ as the null hypothesis, the significance of CRP and L in ROC curve analysis was less than 0.05. It can be seen in the ROC curve that both CRP and $L$ have good diagnostic value for death outcomes. The area under the curve (AUC) of CRP is greater than 0.7 , that is 0.751 , and the area under the curve (AUC) of $L$ is 0.720 . The optimal cutoff values for $C R P$ and $L$ as indicators to determine the outcome of death were calculated using the maximum Youden index, which were 91.5 and 0.615. (See Figure 4)

CT is very important in the clinical diagnosis and typing of COVID-19. This research investigated the CT imaging changes of a patient over 60 years old at different periods, and the results showed that the patient's lung condition gradually improved during the clinical process, and CRP decreased with the improvement of CT imaging. In the above analysis, it was concluded that an increase in CRP index would increase the severity of the disease, which was consistent with CT results. Therefore, in the process of monitoring patients' condition, combining the results of CT imaging to judge the changes of the disease can make the judgment of the condition more accurate. (See Figure 5)

\section{Discussion}

COVID-19 is an acute infectious disease caused by a novel coronavirus (SARS-CoV-2). When SARS-CoV-2 enters the body through the angiotensin-converting enzyme 2 (ACE2) receptor adsorbed on the surface of mucosal epithelial cells, its pathogen-related molecular pattern (PAMP) can be quickly recognized by immune cells and activate the natural immune system to clear the virus, but over activation can cause inflammatory storms ${ }^{\text {[10-11] }}$. The onset of the 
disease is mainly fever, most of which are light, and a few are critical ${ }^{[12-14]}$. Some patients may gradually develop dyspnea. In severe cases, the disease progresses rapidly, and severe inflammatory storms may occur, leading to death, especially in elderly patients.

COVID-19 is a newly discovered human infectious disease ${ }^{[15]}$. The commonly used clinical inflammatory indicators such as CRP and $L$ are helpful in the diagnosis and evaluation of many inflammatory diseases. CRP is an acute temporal protein synthesized by liver cells in response to inflammatory stimuli, such as microbial invasion or tissue damage, in very low concentrations in serum from healthy people $(<5 \mathrm{mg} / \mathrm{L})$, and its concentration significantly increased during bacterial infection or tissue damage ${ }^{[16]}$. $L$ is the core of immune response, which can be divided into three types: T cells, B cells and NK cells. Chaolin $\mathrm{H}$ et al. believed that patients with COVID-19 had a large

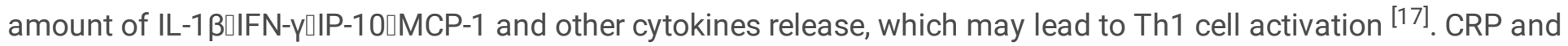
$L$ can be used as sensitive indicators to reflect the control of infection and inflammation. But can these indicators have the same clinical value for COVID-19 patients, especially elderly patients?

The results of this study showed that there was a significant statistical difference between different age groups and outcome of COVID-19 patients, and elderly patients (over 60 years old) were a highly vulnerable group. From the 194 confirmed cases, we extracted 125 samples from the elderly population (over 60 years old), and made a targeted study on the relationship between the demographic and clinical characteristics of the elderly population and admission classification, outcome, and death outcome. The relationship between CRP and admission clinical typing in elderly patients was explored by using bilateral chi-square test or Fisher's exact probabilistic test. The results showed that there was a significant difference between CRP index and clinical typing in the elderly, suggesting that CRP, a laboratory inflammatory index, could be used to assist in determining the severity of COVID-19 in elderly patients.

Furthermore, binary Logistic regression analysis was carried out to investigate whether the elderly patients died or not. The study found that compared with the elderly patients who died, the risk of death was 0.981 times for each unit of decline in CRP, and the 95\% interval of OR value was all less than 1. In the diagnostic relationship between $C R P, L$ and death outcome in elderly patients, we used non-death outcome and death outcome as the basis for positive classification. We took $A U C=0.5$ as the null hypothesis, and the significance of $C R P$ and $L$ in ROC curve analysis was less than 0.05 . It can be seen from the ROC curve that both CRP and $L$ have good diagnostic values for death outcomes, and the optimal thresholds for $L$ and CRP diagnostic death outcomes were determined by the maximum Youden index, which were 91.5 and 0.615 , respectively.

CT is very important in the clinical diagnosis and typing of COVID-19. The results of this study showed that CRP was consistent with CT in judging the changes of the disease, and it could be used in combination to judge the changes of the disease.

To sum up, the results of this clinical study showed that COVID-19 patients over 60 years old had severe clinical classification and poor prognosis. CRP and $L$ can be used as the key monitoring indicators of COVID-19 in elderly patients. Combined with CT examination and observation of its dynamic changes, CRP and L can play an important clinical guiding value in the assessment of disease severity and prognosis.

\section{Declarations}

\section{Ethics approval and consent to participate}


Because this study was a retrospective analysis and all the data were anonymous, we did not involve patients' private information. This study was approved by the Medical Ethics Review Board of Wuhan University of Science and Technology (No. 202009).

\section{Consent for publication}

After revision and review by all authors, all authors finally agree to publish.

\section{Availability of data and materials}

The datasets used and/or analysed during the current study are available from the corresponding author on reasonable request.

\section{Competing interests}

The authors declare that they have no competing interests.

Funding: No funding

\section{Authors' contributions}

$\mathrm{QH}, \mathrm{XFH}, \mathrm{XCX}$, and SYC contributed equally to this article, they are co-first author. The whole experimental design was completed under the guidance of professor QW and professor QMW. QH and XFH were responsible for the arrangement of data, XCX and SYC are responsible for analyzing the data, KWG and XLL participated in the interpretation of the results, QW and WXL wrote the initial draft with all authors providing critical feedback and edits to subsequent revisions. QW, KWG and QMW reviewed and revised the paper before submission, they are cocorresponding authors.

\section{Acknowledgements}

On behalf of the author, I would like to express my heartfelt thanks to the clinicians of Tianyou Hospital of Wuhan University of Science and Technology and the research team of the school of medicine of Wuhan University of Science and Technology for their selfless dedication.

\section{Patient data}

We increased the data sample size on the basis of 132 data samples from another published paper. In another paper, the research point was the judgment value of SAA in the assessment of COVID- 19's disease severity and prognosis. In this paper, CRP and L were emphasized as important indicators of severe coronavirus disease and poor prognosis in elderly patients.

\section{Abbreviations}

COVID-19: Coronavirus disease 2019; CRP: C-reactive protein; L: Lymphocyte; ROC: Receiver operating characteristic; AUC: Area under the curve; CT: Computer tomography; SARS-CoV-2 : Severe Acute Respiratory Syndrome Coronavirus 2; SAA: Serum amyloid A; ICU: Intensive care unit; Cl: Confidence interval; M: Mean; SD: Standard deviations; HOD: Hospitalization days; OR: Odds ratio; ACE2: Angiotensin-converting enzyme 2; PAMP: Pathogenrelated molecular pattern. 


\section{References}

[1] Zhou T, Liu Q, Yang Z, et al. Preliminary prediction of the basic reproduction number of the Wuhan novel coronavirus 2019-nCoV. J Evid Based Med,2020,Feb 12.doi:10.1111/jebm.12376.

[2] Na Zhu, Dingyu Zhang, Wenling Wang,et al. A Novel Coronavirus from Patients with Pneumonia in China.2019. The New England of Journal of Medicine, 2020;382:727-733.

[3] Sun P, Lu X, Xu C, Sun W, Pan B. Understanding of COVID-19 based on current evidence [published online ahead of print, 2020 Feb 25]. J Med Virol. 2020;10.1002/jmv.25722. doi:10.1002/jmv.25722

[4] Lu R, Zhao X, Li J, et al. Genomic characterisation and epidemiology of 2019 novel coronavirus: implications for virus origins and receptor binding. Lancet. 2020;395(10224):565-574. doi:10.1016/S0140-6736(20)30251-8

[5] Wang L, He W, Yu X, et al. Coronavirus Disease 2019 in elderly patients: characteristics and prognostic factors based on 4-week follow-up [published online ahead of print, 2020 Mar 30]. J Infect. 2020;S0163-4453(20)30146-8. doi:10.1016/j.jinf.2020.03.019

[6] Mahase E. Covid-19: death rate is 0.66\% and increases with age, study estimates. BMJ. 2020;369:m1327. Published 2020 Apr 1. doi:10.1136/bmj.m1327

[7] Cao Jianlei., Tu Wen-Jun., Cheng Wenlin., et al. Clinical Features and Short-term Outcomes of 102 Patients with Corona Virus Disease 2019 in Wuhan, China. Clin. Infect. Dis., undefined(undefined), undefined. doi:10.1093/cid/ciaa243

[8] Liu K, Chen Y, Lin R, Han K. Clinical features of COVID-19 in elderly patients: A comparison with young and middle-aged patients [published online ahead of print, 2020 Mar 27]. J Infect. 2020;. doi:10.1016/j.jinf.2020.03.005

[9]Li H, Xiang X, Ren H, et al. Serum Amyloid A is a biomarker of severe Coronavirus Disease and poor prognosis. J Infect. 2020;80(6):646-655. doi:10.1016/j.jinf.2020.03.035

[10]Wan Y, Shang J, Graham R, et al. Receptor recognition by novel coronavirus from Wuhan: An analysis based on decade-long structural studies of SARS[J]. J Virol, 2020, pii: JVI.00127-00120. DOI: 10.1128/JVI.00127-20.

[11] Kowalczuk S, Bröer A, Tietze N, et al. A protein complex in the brush-border membrane explains a Hartnup disorder allele[J].FASEB J, 2008, 22(8): 2880-2887. DOI: 10.1096/fj.08-107300.

[12] Tang N, Li D, Wang X, et al. Abnormal Coagulation parameters are associated with poor prognosis in patients with novel coronavirus pneumonia[J]. J Thromb Haemost, 2020. DOI: 10.1111/jth.14768.

[13] Huang C, Wang Y, Li X, et al. Clinical features of patients infected with 2019 novel coronavirus in Wuhan,China.Lancet,2020,395(10223):497-506.

[14] Wang D, Hu B, Hu C, et al .Clinical Characteristics of 138 Hospitalized Patients With 2019 Novel CoronavirusInfected Pneumonia in Wuhan,China.JAMA,2020,Feb 7.doi:10.1001/jama.2020.1585.

[15] Yin Y, Wunderink RG. MERS, SARS and other coronaviruses as causes of

pneumonia. 2018; 23(2): 130-137

Page $11 / 15$ 
[16] Deme D, Telekes A. A C-reaktív protein (CRP) plazmaszintjének prognosztikai jelentősége az onkológiában [Prognostic importance of plasma C-reactive protein (CRP) in oncology]. Orv Hetil. 2017;158(7):243-256.

doi:10.1556/650.2017.30646

[17] Huang C, Wang Y, Li X, et al. Clinical features of patients infected with 2019 novel coronavirus in Wuhan, China[J].Lancet,2020,395(10223):497-506.

\section{Figures}
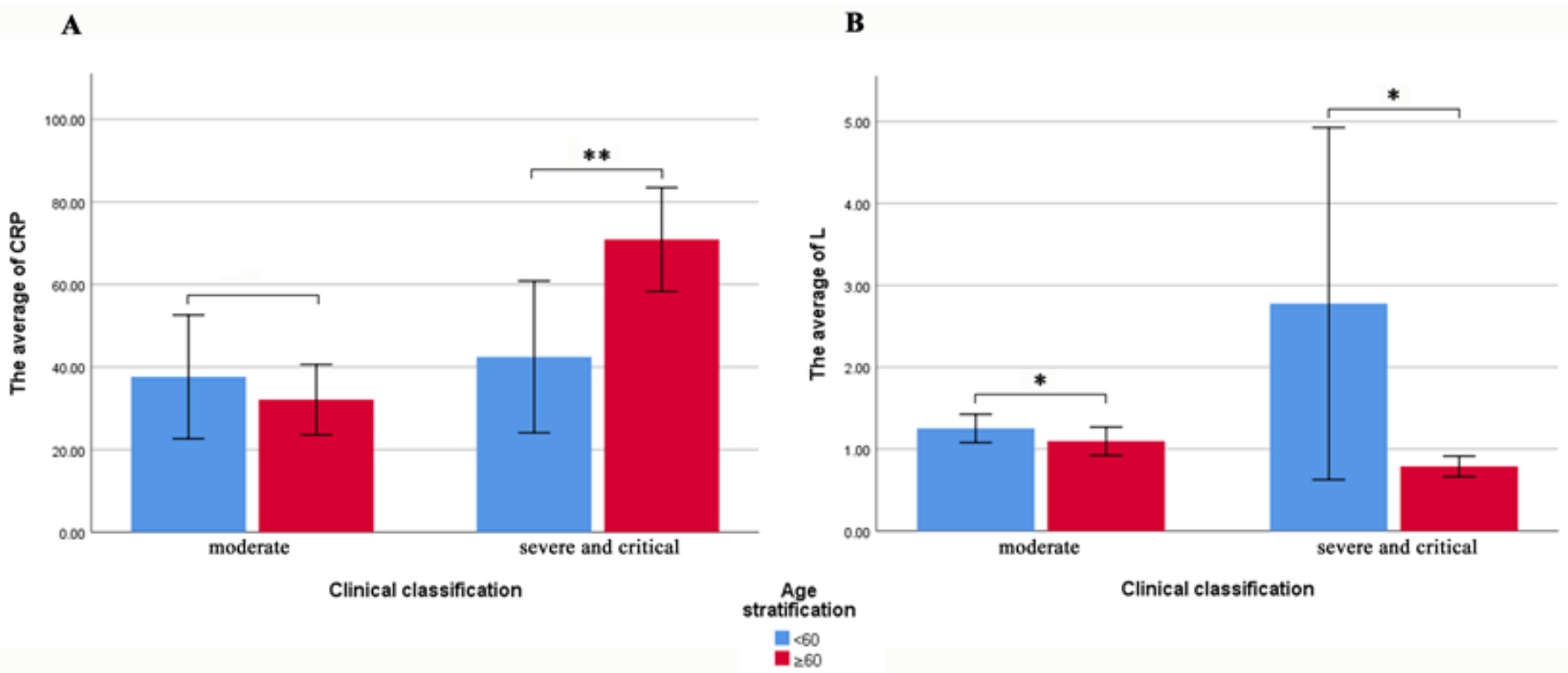

Note: ${ }^{*}$ means $\mathrm{p}<0.05$, ** means $\mathrm{p}<0.01$; Normal reference values: CRP $(<3 \mathrm{mg} / \mathrm{L}) ; \mathrm{L}\left(1.1 \times 10^{9} / \mathrm{L}-3.2 \times 10^{9}\right.$ / L)

\section{Figure 1}

Analysis of differences in clinical typing between patients aged $\geq 60$ years and $<60$ years. Figure $1 \mathrm{~A}$. The length of the bar indicates the average level of CRP; Figure1B. The length of the bar indicates the average count of L. * means $p<0.05$, ** means $p<0.01$; Normal reference values: CRP (<3mg / L); L $(1.1 \times 109 / \mathrm{L}-3.2 \times 109$ / L) 
A

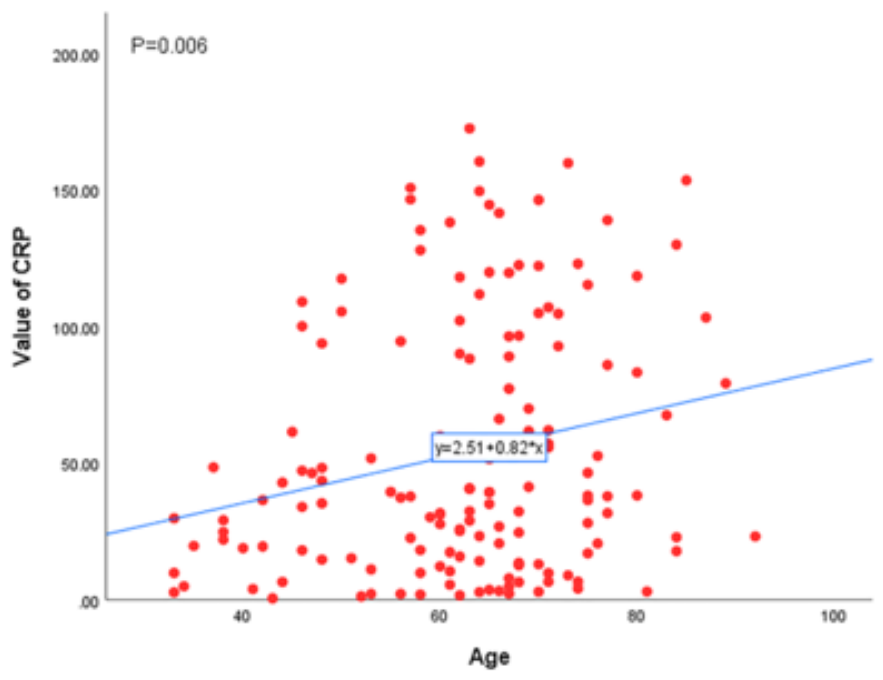

B

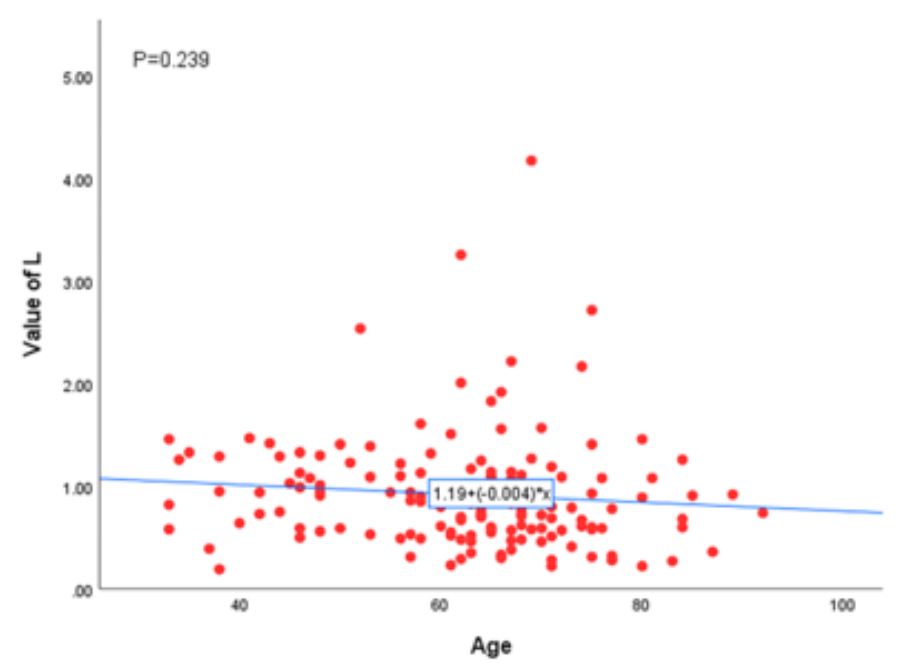

Note: CRP (<3mg / L $)$; L $\left(1.1 \times 10^{9} / \mathrm{L}-3.2 \times 10^{9} / \mathrm{L}\right)$.

\section{Figure 2}

The regression line between CRP level/L count and age in COVID-19 patients Figure2A. The X-axis corresponding to each point is the patient's age, and the Y-axis is the value of CRP. The regression line is obtained by ordinary least squares method. Figure2B. The X-axis corresponding to each point is the patient's age, and the $\mathrm{Y}$-axis is the value of L. The regression line is obtained by ordinary least squares method.

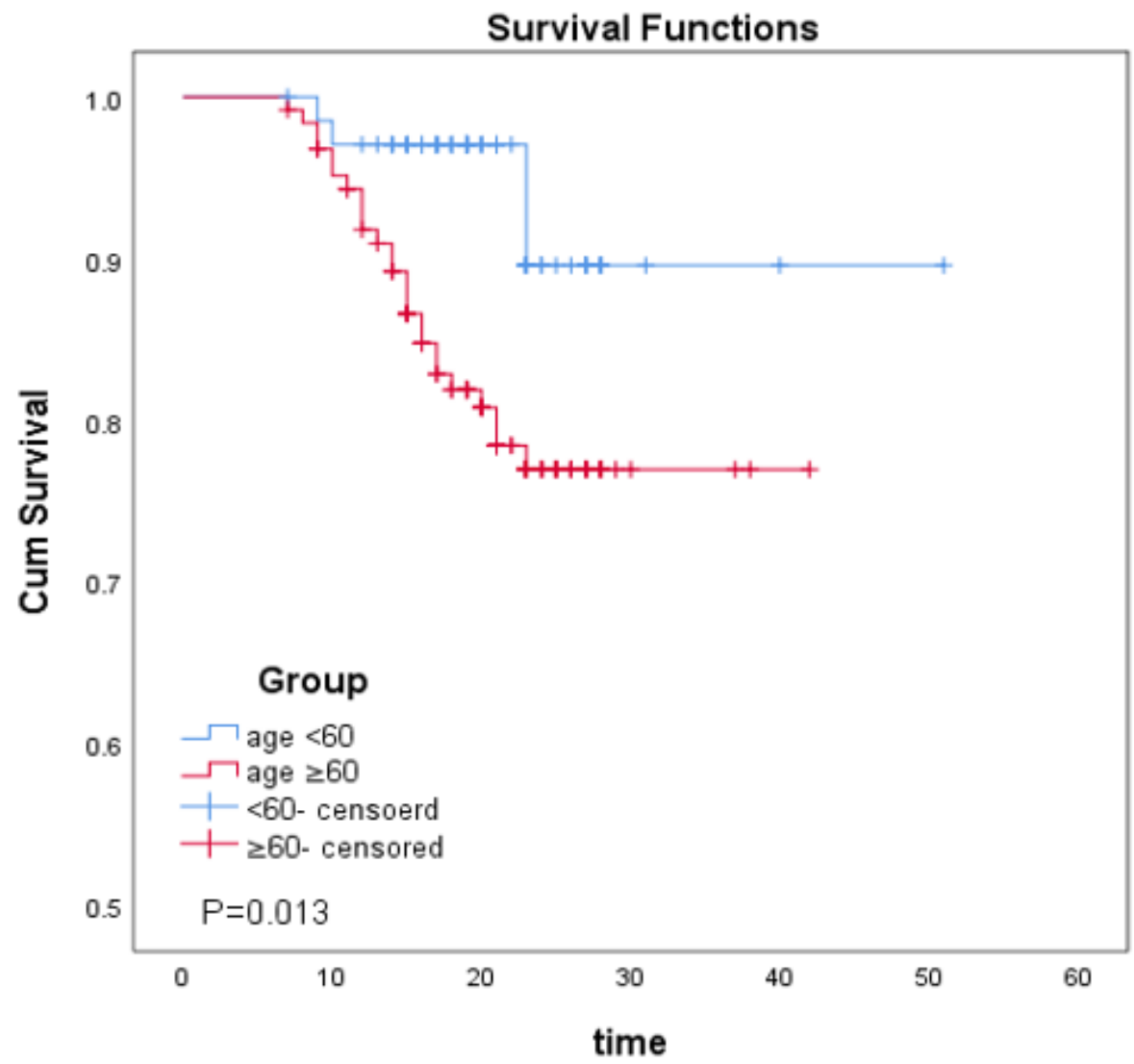




\section{Figure 3}

Survival analysis among patients aged $\geq 60$ years and $<60$ years Kaplan-Meier curve demonstrating survival of COVID-19 patients. $P$ values for survival analysis are derived by the log-rank test.

$\mathbf{A}$

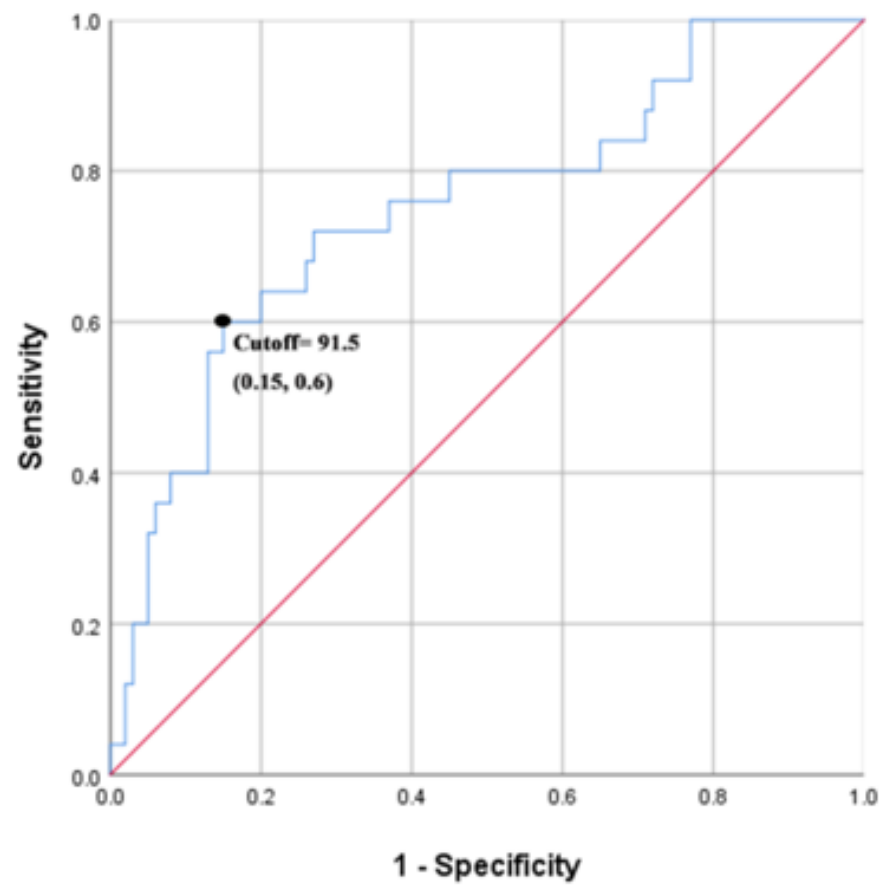

B

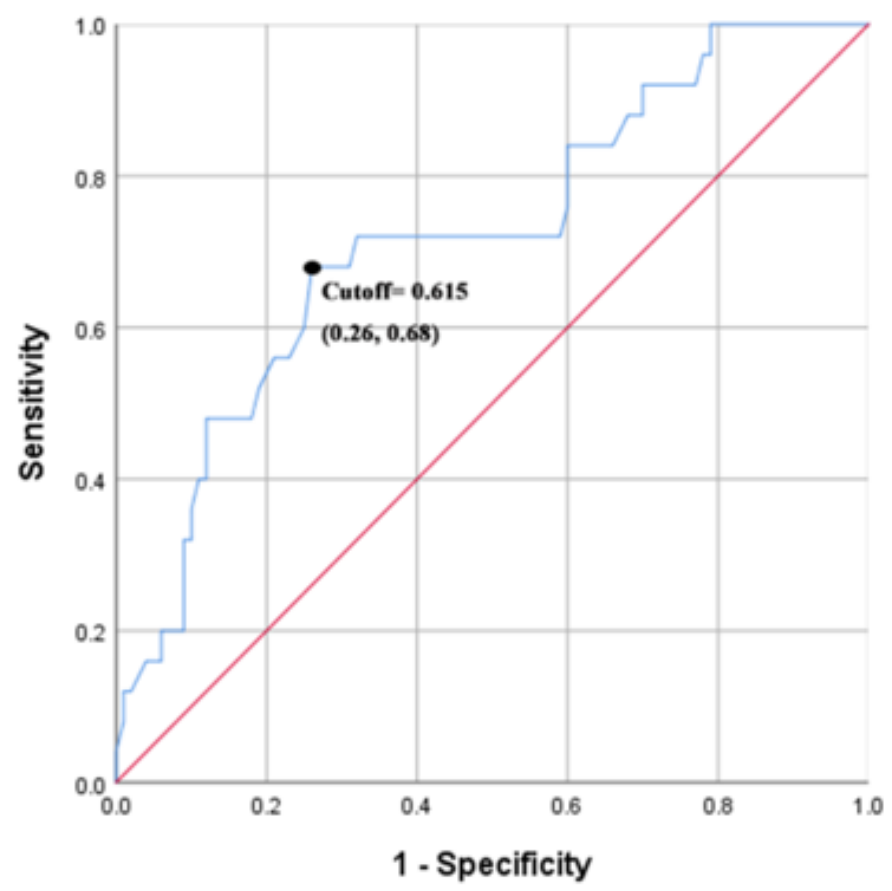

Figure 4

The prognostic value of CRP and $L$ in elderly patients with COVID-19 Figure4A. ROC curve: CRP's judgment on the prognosis of elderly COVID-19 patients. Figure4B. ROC curve: L's judgment on the prognosis of elderly COVID-19 patients. The optimal cutoff values for CRP and $L$ were calculated using the maximum Youden index. 


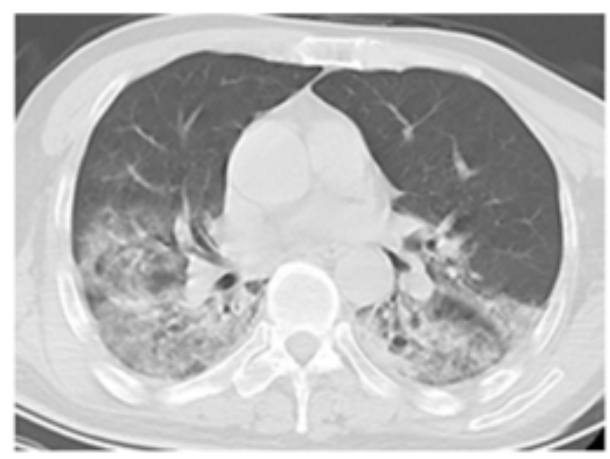

2020.2.1.Lung CT imaging findings: large sheet ground glass shadow and consolidation shadow were seen in both lungs, and air bronchi sign was seen inside.CT score is 3 points.

2020.2.1 CRP in peripheral blood was $139.10 \mathrm{mg} / \mathrm{L}$ (normal reference value: $0-3 \mathrm{mg} / \mathrm{L})$.

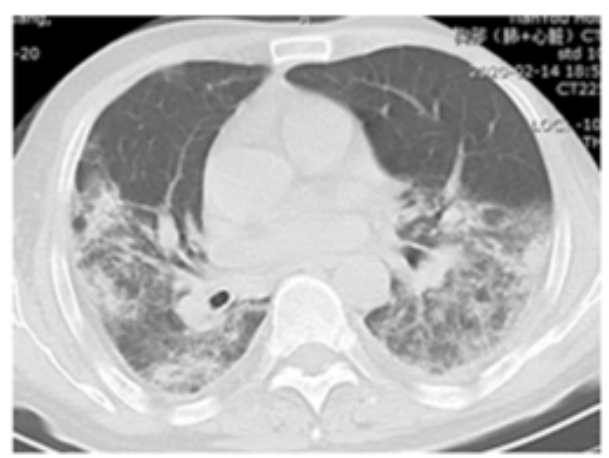

2020.2.1.Lung CT imaging findings: the range of bilateral lung lesions was slightly larger than before, and the density of some lesions was slightly reduced.CT score is 3 points. 2020.2.14 CRP in peripheral blood was $54.1 \mathrm{mg} / \mathrm{L}$ (normal reference value: $0-3 \mathrm{mg} / \mathrm{L})$.

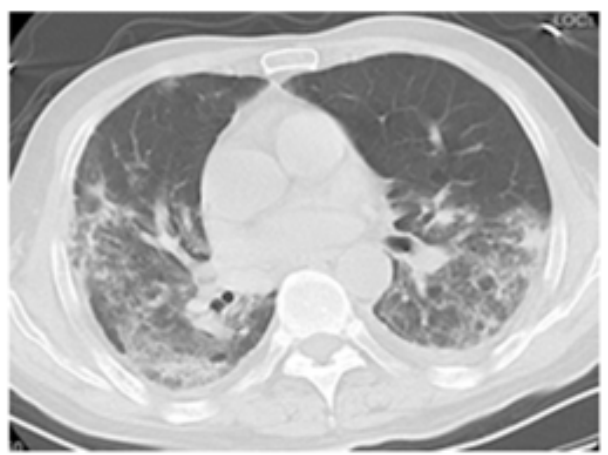

2020.2.20.Lung CT imaging findings: the bilateral lung lesions were slightly smaller than the previous range, with reduced density and cordage.CT score is 2 points. 2020.2.20 The CRP in peripheral blood was $26.7 \mathrm{mg} / \mathrm{L}$ (normal reference value: $0-3 \mathrm{mg} / \mathrm{L}$ ).

\section{Figure 5}

(A male patient aged over 60 years): serial CT scans and CRP dynamic changes 